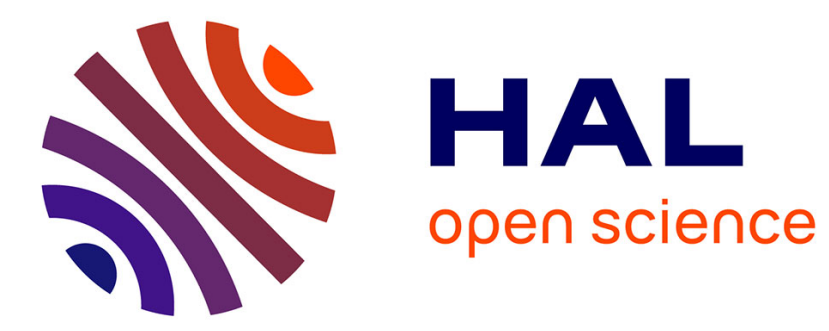

\title{
Pharmacophore modelling of 17 $\beta$-HSD1 enzyme based on active inhibitors and enzyme structure
}

\author{
Sampo Karkola, Sari Alho-Richmond, Kristiina Wähälä
}

\section{To cite this version:}

Sampo Karkola, Sari Alho-Richmond, Kristiina Wähälä. Pharmacophore modelling of 17 $\beta$-HSD1 enzyme based on active inhibitors and enzyme structure. Molecular and Cellular Endocrinology, 2009, 301 (1-2), pp.225. 10.1016/j.mce.2008.08.030 . hal-00532071

\section{HAL Id: hal-00532071 \\ https://hal.science/hal-00532071}

Submitted on 4 Nov 2010

HAL is a multi-disciplinary open access archive for the deposit and dissemination of scientific research documents, whether they are published or not. The documents may come from teaching and research institutions in France or abroad, or from public or private research centers.
L'archive ouverte pluridisciplinaire $\mathbf{H A L}$, est destinée au dépôt et à la diffusion de documents scientifiques de niveau recherche, publiés ou non, émanant des établissements d'enseignement et de recherche français ou étrangers, des laboratoires publics ou privés. 


\section{Accepted Manuscript}

Title: Pharmacophore modelling of $17 \beta-H S D 1$ enzyme based on active inhibitors and enzyme structure

Authors: Sampo Karkola, Sari Alho-Richmond, Kristiina Wähälä

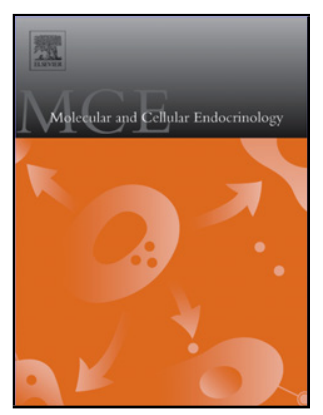

PII:

DOI:

Reference:

To appear in:

Received date:

Revised date:

Accepted date:
S0303-7207(08)00388-2

doi:10.1016/j.mce.2008.08.030

MCE 6961

Molecular and Cellular Endocrinology

29-6-2008

20-8-2008

25-8-2008

Please cite this article as: Karkola, S., Alho-Richmond, S., Wähälä, K., Pharmacophore modelling of 17 $\beta$-HSD1 enzyme based on active inhibitors and enzyme structure, Molecular and Cellular Endocrinology (2007), doi:10.1016/j.mce.2008.08.030

This is a PDF file of an unedited manuscript that has been accepted for publication. As a service to our customers we are providing this early version of the manuscript. The manuscript will undergo copyediting, typesetting, and review of the resulting proof before it is published in its final form. Please note that during the production process errors may be discovered which could affect the content, and all legal disclaimers that apply to the journal pertain. 
Pharmacophore modelling of 17ß-HSD1 enzyme based on active inhibitors and enzyme structure

Sampo Karkola, Sari Alho-Richmond and Kristiina Wähälä*

Laboratory of Organic Chemistry, Department of Chemistry, PO Box 55, FIN-00014 University of Helsinki, Helsinki, Finland

*Corresponding author: Prof. Kristiina Wähälä, Laboratory of Organic Chemistry, Department of Chemistry, PO Box 55, FIN-00014 University of Helsinki, Finland. Tel: +358 9 19150356, Fax: +358 9 19150357, email: kristiina.wahala@helsinki.fi 
Keywords

17 $\beta$-hydroxysteroid dehydrogenase, pharmacophore, 3D database screening, docking

\begin{abstract}

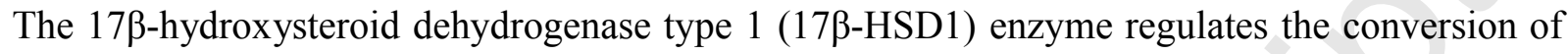
estrone (E1) to the biologically active estradiol (E2). Due to its role as a key enzyme in female hormone production, it has emerged as an attractive drug target for inhibitor development in relation to hormone-dependent breast cancer. Herein we report four pharmacophore models of $17 \beta$-HSD1 based on a crystal structure, a relaxed crystal structure, a library of $17 \beta$-HSD1 inhibitors and on a docked complex of 17ßHSD1 enzyme and a potent inhibitor. The models were used in screening two databases, which produced novel compounds to be used as leads in our drug design project. The results were validated by docking the compounds to the active site of the 17ß-HSD1 enzyme. With the help of our 3D-QSAR model, these results will be used to develop new inhibitors of $17 \beta$-HSD1 as drug candidates.
\end{abstract}

Introduction

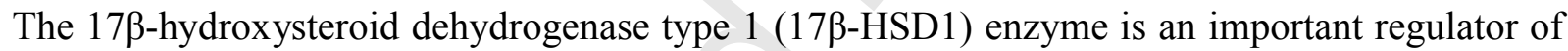
the female hormone production catalysing the NADPH dependent reduction of estrone E1 to the biologically active 17ß-estradiol E2. The enzymatic complex consists of the enzyme and a cofactor, providing the reducing hydride to the reaction. In the active site the conversion from E1 to E2 is performed by a catalytic tetrad consisting of residues Tyr155, Lys159, Ser142 and Asn114 and the cofactor NADPH (Joernvall et al. 1995). Several crystal structures of 17ß-HSD1 with estrogens, androgens and steroid-based inhibitors are available (Azzi et al. 1996, Breton et al. 1996, Gangloff et al. 2002, Ghosh et al. 1995, Han et al. 2000, Sawicki et al. 1999, Shi \& Lin 2004).

Pharmacophore modelling is a new approach in attempts to identify novel scaffolds for $17 \beta$ HSD1 inhibition. A recent paper describes two pharmacophore models based on two crystal structures, one with steroidal ligand equilin (PDB entry code 1EQU, Sawicki et al. 1999) and one with a hybrid inhibitor, occupying both the substrate and the cofactor binding sites (PDB entry code 1I5R, (Qiu et al. 2002, Schuster et al. 2008)). As a result from database searching, four compounds with $\mathrm{IC}_{50}$ values below $50 \mu \mathrm{M}$ were identified.

Parallel to our study on 3D-QSAR model of $17 \beta$-HSD1 inhibitors, we generated four pharmacophore models based on A) $17 \beta$-HSD1 crystal structure which was relaxed using molecular dynamics simulations, B) our thienopyrimidinone inhibitor library, C) 17 $\beta$-HSD1 
crystal structure with bound estradiol (PDB entry code 1FDT, flexible loop conformation B)(Breton et al. 1996)), and D) the interactions of our most potent inhibitor 1 and the enzyme (Karkola et al. 2008). These pharmacophore models were evaluated and compared using database screening and molecular docking to find a model which can be used in discovering novel lead compounds. Pharmacophore models are very useful in 3D-database search and in finding novel scaffolds for inhibitors. Despite of the long history of 17 $\beta$-HSD1 research, pharmacophore models have only recently emerged as a valuable tool for drug discovery discovery.

\section{Methods}

Pharmacophores A and B were generated using Catalyst protocols implemented in Discovery Studio 2.1 (Accelrys, Inc.). Pharmacophores C and D were generated with the automated pharmacophore generation program LigandScout 2.02 (Wolber et al. 2006, Wolber \& Langer 2005). The commercial database MayBridge HitFinder Collection database (Maybridge, UK) consisting of 14400 compounds was screened using the 3D Database Search implemented in Discovery Studio. The dockings were made with GOLD Suite (Verdonk et al. 2003). All the protocols mentioned in the text are implementations of Discovery Studio 2.1 unless otherwise indicated.

The relaxed crystal structure of $17 \beta$-HSD1 from molecular dynamics simulations (Karkola et al. 2008) was used to find the pharmacophoric features that are important for the ligand binding in the absence of crystal packing effects. The Interaction Generation protocol was used to identify hydrogen bond acceptor, donor and hydrophobic features inside the active site. These features were then clustered and the most representative features were selected and included in the pharmacophore hypothesis. Exclusion spheres were manually placed on residues flanking the active site (pharmacophore $\mathrm{A}$ ).

The inhibitor alignment from our 3D-QSAR study (Karkola et al. 2008) was used in the Common Feature Pharmacophore Generation protocol to generate a pharmacophore hypothesis in the absence of a receptor. The inhibitors were classified based on their activity using the Principal property in Discovery Studio. A value of 2 was assigned to inhibitors having the LOGIT transformed $\mathrm{pIC}_{50}$ value over 7 , a value of 1 to inhibitors with a $\mathrm{pIC}_{50}$ value between 6-7 and 0 to inhibitors with a $\mathrm{pIC}_{50}$ value below 6 . This classification is used by the protocol to distinguish active, moderately active and inactive inhibitors. Hydrogen bond acceptor, donor and hydrophobic features were generated. Ten pharmacophore hypotheses were produced and the best one was selected for further studies (pharmacophore B).

Because Discovery Studio lacks a tool that generates a pharmacophore based on the interactions between a bound ligand and a protein in a crystal structure, we used the LigandScout program. In 
LigandScout, the interactions between a ligand and a protein are converted to a pharmacophore. However, LigandScout produces multiple features (acceptor and donor) for eg. hydroxyl groups. These features have to be simplified, either manually or automatically, before database screening in Catalyst. The simplified pharmacophore is exported as a hypoedit script and converted to Catalyst format with the Hypoedit tool (Accelrys, Inc.). The crystal structure 1FDT (Breton et al. 1996) was downloaded from the Protein Data Bank (Berman et al. 2003) and the pharmacophore was automatically generated. The LigandScout pharmacophore was exported and converted to Catalyst pharmacophore query (pharmacophore C).

In order to widen the diversity of the pharmacophores of $17 \beta$-HSD1 enzyme, the interactions between the most potent inhibitor 1 in our 3D-QSAR study (Karkola et al. 2008), and the enzyme were used in pharmacophore generation. Again, LigandScout was used for automated pharmacophore generation and the hypothesis was converted to Catalyst query (pharmacophore D).

\section{The Maybridge Database}

The Maybridge HitFinder Database (Maybridge) was downloaded and the structures were subjected to Diverse Conformation Generation protocol to produce a maximum of 10 conformations for each compound in the database. The resulting conformers were used in 3D Database Search protocol. The Ligand Pharmacophore Mapping protocol was used to rank the conformers from the database based on their fit to the pharmacophores. This protocol was used to identify the best matching conformer from each compound which was then exported for further studies.

Results and discussion

The pharmacophores and database screening

The Interaction Generation for the relaxed crystal structure and subsequent clustering of the generated interactions produced two hydrogen bond donor and one acceptor features (Pharmacophore A, Figure 1 A). Donors were pointed towards Ser222 and Glu282 and the acceptor was pointed towards Ser142. Two hydrophobes were located in the center of the active site. The Maybridge HitFinder Collection database with generated conformers was searched to find compounds that fulfil the pharmacophoric requirements. As a result, 447 conformers (representing 138 compounds) were exported and ranked based on Ligand Pharmacophore Mapping protocol. Further refining was made based on FitValue, which measures the quality of the mapping. All compounds having a scaled FitValue (range from 0 to 1) less than 0.5 were rejected, leaving 20 compounds. To validate the database search and the mapping, these compounds were docked with GOLD Suite (Goldscore scoring function) and rescored with 
Chemscore scoring function. The most promising compounds were selected based on an overall consensus of the FitValue, Goldscore ranking and Chemscore ranking. The compounds expected to have 17ß-HSD1 inhibitory activity are shown in Figure 2. In comparison, a compound similar to the first structure in Figure 2, Pharmacophore A, but having a propyl ring instead of the fluoro-substituted aryl ring gave a scaled FitValue less than 0.5 showing the importance of the fluoro-substituted aryl ring.

The Common Feature Pharmacophore Generation protocol for the aligned thienopyrimidinone inhibitors produced three hydrogen bond donor features located at both carbonyl groups and the pyrimidinone nitrogen of the most potent inhibitor 1 (Pharmacophore B, Figure 1 B). The acceptor feature at the pyrimidinone nitrogen was changed to a hydrophobic feature due to the absence of a hydrogen bond donor at that position in the active site. Two other hydrophobes were located near the thiophenyl substituent and the partially unsaturated ring A of the inhibitor 1, Figure 2. Ten exclusion spheres were located around the inhibitors. An identical protocol in database screening, mapping and ranking was used as for the Pharmacophore A. The screening produced 667 conformers (representing 274 compounds) and the ranking after mapping gave 19 compounds. The docking and consensus evaluation of the FitValue, Goldscore and Chemscore rankings resulted in the compounds shown in Figure 2. It is expected that these compounds share a similar binding mode than the potent inhibitor 1 . Therefore, we predicted the $\mathrm{IC}_{50}$ values for these compounds based on our CoMFA model (Karkola et al. 2008). The predicted values were in sub-micromolar level for these compounds.

The automated pharmacophore generation for the crystal structure 1FDT from LigandScout resulted in two hydrogen bond acceptor and two hydrophobic features after automatic simplification (Pharmacophore C, Figure $1 \mathrm{C}$ ). The acceptors were pointed from the hydroxyl groups of E2 to Tyr155 and His221. It should be mentioned, that the acceptor pointing towards His221 can also be a donor feature depending on the tautomerism of His221. The two hydrophobes were located at C18 methyl group and the aromatic ring A of E2. A tight spatial restriction was generated with the Exclusion Volume Coat generation giving 29 exclusion spheres around E2. The following screening (726 conformers), mapping (274 compounds), ranking (9 compounds), docking and consensus evaluation resulted in compounds shown in Figure 2. Compared to the pharmacophore model based on the crystal structure 1equ (Schuster et al. 2008), which has a donor pointing to His 221 and an acceptor pointing to Ser142, LigandScout generated a donor interaction to His221 and an acceptor to Tyr155 in addition to two hydrophobic centres. This is, of course, due to the usage of a different crystal structure. As the results from a database screening using the 1equ model (Schuster et al. 2008) were not reported, it is difficult to compare the two models based on the crystal structures.

LigandScout was also used for the pharmacophore generation of the complex between the most potent inhibitor 1 and 17ß-HSD1. As a result, one hydrogen bond donor feature towards the 
backbone carbonyl of Gly144, two acceptor features towards Tyr218 and Arg258, and one hydrophobic feature around the thiophenyl substituent were generated (Pharmacophore D, Figure1 D). The subsequent database screening (18 conformers), mapping (3 compounds) and docking resulted in compounds shown in Figure 2. One of these compounds (2) has already been shown to possess antitumour activity (Berrut et al. 2005). The $\mathrm{IC}_{50}$ values for these compounds were predicted based on our CoMFA model (Karkola et al. 2008), because of the expected similar biding mode compared to the inhibitor 1 . The CoMFA model predicted sub-micromolar $\mathrm{IC}_{50}$ values for these compounds.

The compounds shown in Figure 2 are expected to have inhibitory activity against the 17ß-HSD1 enzyme. It should be noted, however, that among the compounds there are several functional groups (nitro, thiol and sulfonyl) that may cause unwanted effects during metabolism. Therefore, these compounds are to be treated as lead compounds and further modification should be conducted to finally end up with drug candidates. Additionally, not all compounds fulfil all the pharmacophoric requirements. This is due to the selection of the compounds having the scaled FitValue less than 1.0. The hits found for each of the pharmacophores are different, because no two pharmacophores share enough similar features, either in number or in property. Therefore, the Mapping and ranking (in terms of the FitValue) procedure gives only compounds that fulfil the pharmacophore in question.

\section{Conclusions}

We have generated four different pharmacophore models for the 17ß-HSD1 enzyme and screened a commercial database to find possible lead compounds for our drug discovery project. We identified compounds that fit well to the active site of 17 $\beta$-HSD1 in terms of pharmacophore mapping and docking using two different scoring functions. The activities of some of the compounds were predicted based on our CoMFA model showing very promising inhibitory activity. Altogether, we have shown that pharmacophore modelling, database screening and prediction of the compounds with docking and QSAR model have resulted in truly promising compounds for our drug discovery project. 
Figures

Figure 1. The pharmacophores A, B, C and D. Hydrogen bond donors are coloured magenta, acceptors are coloured green, hydrophobes are coloured cyan and the exclusion spheres are coloured grey. The interacting residues, estradiol and the inhibitor $\mathbf{1}$ are represented for clarity. Only selected exclusion spheres in pharmacophore D are displayed for clarity.
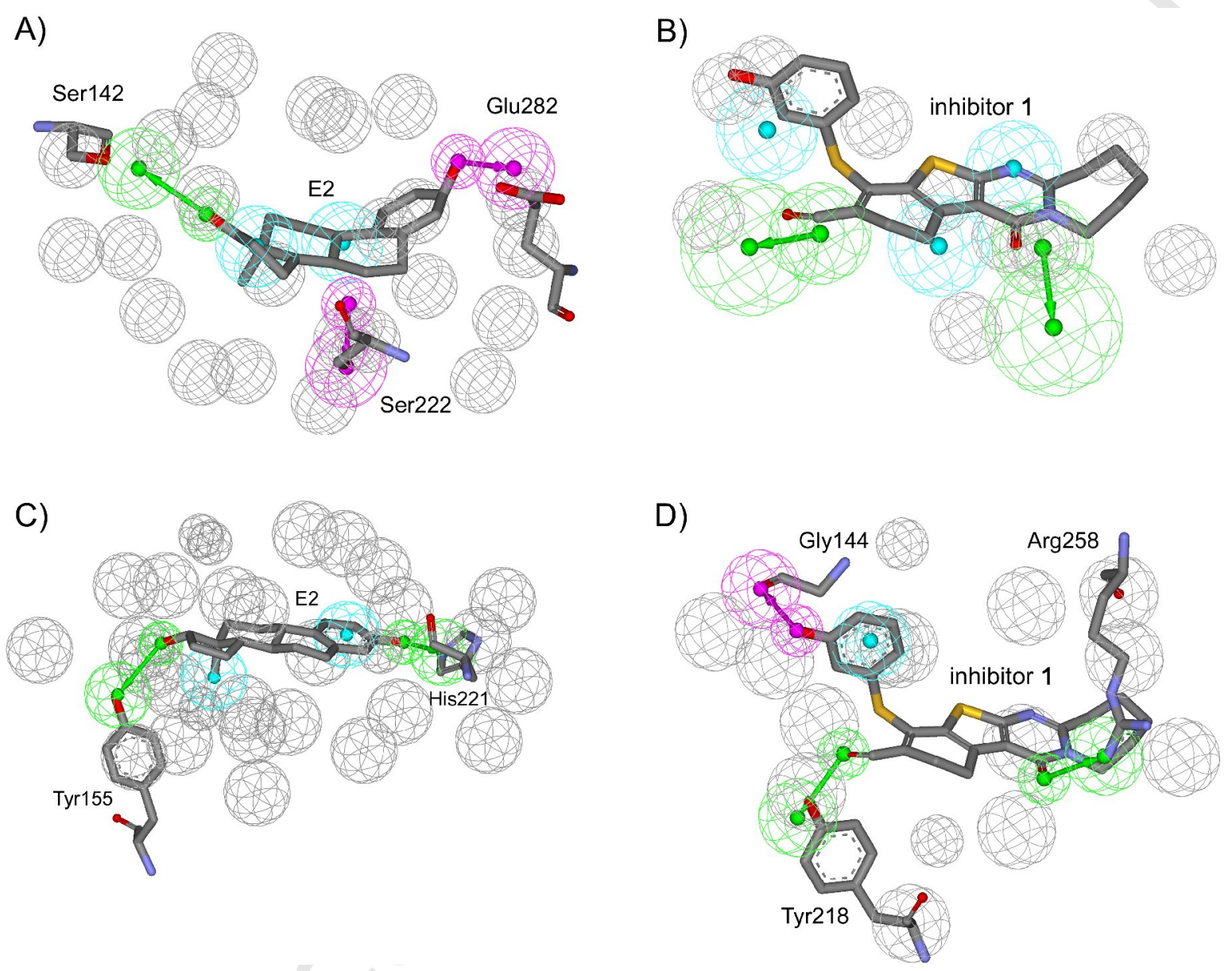
Figure 2. The compounds matching the pharmacophores, and validated by docking and QSAR prediction and the most potent inhibitor 1 with ring labelling shown.

Pharmacophore A<smiles>Cc1noc(C)c1C(=O)ON=C(N)Cc1ccc(-c2csnn2)cc1</smiles>

\section{Pharmacophore B}<smiles>Cc1cc(C)n(-c2ccc(Sc3ccc(Cl)cc3)nn2)c1</smiles><smiles>CCCCOc1cc(C(F)(F)F)nc2ccc(CSc3nc4ccccc4s3)cc12</smiles><smiles>Cc1ccc(S(=O)(=O)Nc2ccc(-n3cc(C(F)(F)F)cn3)cc2)cc1</smiles>

Pharmacophore C<smiles>Cc1nnsc1C(=O)Nc1ccc2c(c1)OCCO2</smiles><smiles>Cc1cc(C(F)(F)F)ccc1C(=O)Sc1ccc(F)cc1</smiles><smiles>Cn1c(SCc2ccc(C(F)(F)F)cc2)nnc1C(F)(F)F</smiles>

Pharmacophore D<smiles>COc1ccccc1C1CCN(c2ccc(CO)cc2[N+](=O)[O-])CC1</smiles><smiles>Nc1ccc(Oc2ccc(C(F)(F)F)cc2)nc1</smiles><smiles>Cn1c(S)nnc1-c1ccc(OCC(F)(F)F)nc1</smiles>

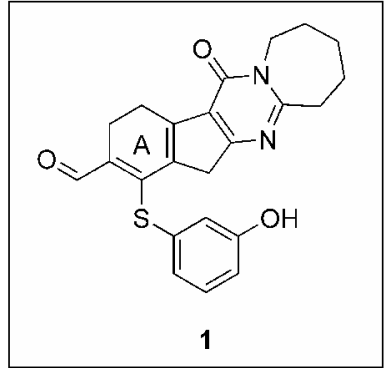




\section{References}

Accelrys Inc. Discovery Studio 2.1, 10188 Telesis Court,Suite 100 San Diego,CA 92121,USA.

Azzi, A., Rehse, P.H., Zhu, D., Campbell, R.L., Labrie, F., Lin, S. 1996. Crystal structure of human estrogenic 17ßhydroxysteroid dehydrogenase complexed with 17ß-estradiol. Nat. Struct. Mol. Biol. 3, 665-668.

Berman, H.M., Henrick, K., Nakamura, H. 2003. Announcing the worldwide Protein Data Bank. Nat. Struct. Biol. 10,980

Berrut, S., Dimarq, J.L., Guenneugues, M. 2005. New norcantharidin derivatives e.g. used for treating cancer, neurodegenerative diseases, restenosis, diabetes, asthma and bacterial and fungal infections. Patent WO2005037839

Breton, R., Housset, D., Mazza, C., FontecillaCamps, J.C. 1996. The structure of a complex of human 17 $\beta-$ hydroxysteroid dehydrogenase with estradiol and $\mathrm{NADP}^{+}$identifies two principal targets for the design of inhibitors. Structure 4, 905-915.

Gangloff, A., Shi, R., Nahoum, V., Lin, S. 2002. Pseudo-symmetry of C19-steroids, alternative binding orientations and multispecificity in human estrogenic 17ß-hydroxysteroid dehydrogenase. FASEB J. 17, 274-276.

Ghosh, D., Pletnev, V.Z., Zhu, D., Wawrzak, Z., Duax, W.L., Pangborn, W., Labrie, Fernand and Lin,ShengXiang 1995. Structure of human estrogenic $17 \beta$-hydroxysteroid dehydrogenase at $2.20 \AA$ resolution. Structure 3, 503513.

Han, Q., Campbell, R.L., Gangloff, A., Huang, Y., Lin, S. 2000. Dehydroepiandrosterone and Dihydrotestosterone Recognition by Human Estrogenic 17ß-Hydroxysteroid Dehydrogenase. C-18/C-19 Steroid Discrimination and Enzyme-Induced Strain. J. Biol. Chem. 275, 1105-1111.

Joernvall, H., Persson, B., Krook, M., Atrian, S., Gonzalez-Duarte, R., Jeffery, J., Ghosh, D. 1995. Short-chain dehydrogenases/reductases (SDR), Biochemistry 34, 6003-6013.

Karkola, S., Lilienkampf, A., Wähälä, K. 2008. A 3D QSAR Model of 17ß-HSD1 Inhibitors Based on a Thieno[2,3- $d$ ]pyrimidin-4(3H)-one Core Applying Molecular Dynamics Simulations and Ligand-Protein Docking. ChemMedChem 3, 461-472.

Maybridge Inc., Trevillet, Tintagel, Cornwall PL34 0HW, UK, www.maybridge.com.

Qiu, W., Campbell, R.L., Gangloff, A., Dupuis, P., Boivin, R.P., Tremblay, M.R., Poirier, D., Lin, S. 2002. A concerted, rational design of type 1 17 $\beta$-hydroxysteroid dehydrogenase inhibitors: estradiol-adenosine hybrids with high affinity. FASEB J. 16, 1829-1831.

Sawicki, M.W., Erman, M., Puranen, T., Vihko, P., Ghosh, D. 1999. Structure of the ternary complex of human 17 $\beta$-hydroxysteroid dehydrogenase type 1 with 3-hydroxyestra-1,3,5,7-tetraen-17-one (equilin) and NADP+. Proc. Natl. Acad. Sci. 96, 840-845. 
Schuster, D., Nashev, L.G., Kirchmair, J., Laggner, C., Wolber, G., Langer, T., Odermatt, A. 2008. Discovery of Nonsteroidal 17 $\beta$-Hydroxysteroid Dehydrogenase 1 Inhibitors by Pharmacophore-Based Screening of Virtual Compound Libraries. J. Med. Chem. DOI:10.1021/jm800054h

Shi, R., Lin, S. 2004. Cofactor Hydrogen Bonding onto the Protein Main Chain Is Conserved in the Short Chain Dehydrogenase/Reductase Family and Contributes to Nicotinamide Orientation. J. Biol. Chem. 279, 1677816785.

Verdonk, M.L, Cole, J.C., Hartshorn, M.J., Murray, C.W., Taylor, R.D. 2003. Improved protein-ligand docking using GOLD. Proteins 52, 609-623.

Wolber, G., Dornhofer, A.A., Langer, T. 2006. Efficient overlay of small organic molecules using 3D pharmacophores. J. Comput. Aided Mol. Des. 20, 773-788.

Wolber, G., Langer, T. 2005. LigandScout: 3-D Pharmacophores Derived from Protein-Bound Ligands and Their Use as Virtual Screening Filters. J. Chem. Inf. Mol. 45, 160-169. 